21st Particles and Nuclei International Conference (PANIC 2017)

International Journal of Modern Physics: Conference Series

Vol. 46 (2018) 1860005 (8 pages)

(C) The Author(s)

DOI: $10.1142 / \mathrm{S} 2010194518600054$

\title{
Experimental Review of Hadron Spectroscopy
}

\author{
Chengping Shen \\ School of Physics and Nuclear Energy Engineering, \\ Beihang University, Beijing 100191, China \\ shencp@buaa.edu.cn \\ Suxian Li \\ School of Physics and Nuclear Energy Engineering, \\ Beihang University, Beijing 100191, China
}

Published 3 May 2018

\begin{abstract}
Since the invention of the quark model in 1964 hadrons are formed from a quarkantiquark pair called mesons or three quarks called baryons. However, QCD-motivated models for hadrons predict more complex structures on the hadrons components called generically exotics. These include tetraquark, pentaquark, the six-quark H-dibaryon, hybrid, and glueball mesons. Exotic hadrons have been systematically searched for in many experiments and studied in theories. In the past decade, lots of new hadrons that cannot fit into the normal mesons or baryons were discovered, the so-called $X Y Z$ states. Even so, no unambiguous candidates for any of those exotic configurations have been identified. This review presents an overview of the remarkable progress in the field of exotic hadrons over the past few years.
\end{abstract}

Keywords: Exotic hadrons; $X Y Z$ states; Hadron spectroscopy.

\section{Introduction}

During the past fourteen years many charmoniumlike and bottomoniumlike states, the so-called " $X Y Z$ " particles, have been reported in experiments by studying of $B$ decays, initial state radiation, double charmonium production, bottomonia decays, two-photon process, etc ${ }^{1}$. Most of them cannot be described well by quarkonium potential models and may be the good candidates of exotic states. Their unusual properties have stimulated considerable theoretical interest and various interpretations have been proposed, including tetraquarks, molecules, hybrids, or hadrocharmonia.

The results reviewed here come from experiments that operate at vastly different energies including BESIII, LHC experiments, BaBar, Belle and so on.

This is an Open Access article published by World Scientific Publishing Company. It is distributed under the terms of the Creative Commons Attribution 4.0 (CC-BY) License. Further distribution of this work is permitted, provided the original work is properly cited. 


\section{The $X$ states}

The state $X(1835)$ was first observed by the BESII experiment as a peak in the $M\left(\eta^{\prime} \pi^{+} \pi^{-}\right)$spectrum in $J / \psi$ radiative decays ${ }^{2}$. This observation was later confirmed by BESIII with the measured mass of $\left(1836.5 \pm 3_{-2.1}^{+5.6}\right) \mathrm{MeV} / c^{2}$ and width of $\left(190 \pm_{-36}^{+38}\right) \mathrm{MeV}^{3}$; the $X(1835)$ was also observed in the $\eta K_{S}^{0} K_{S}^{0}$ system with its spin parity determined to be $J^{P}=0^{-}$by a partial wave analysis ${ }^{4}$. An anomalously strong enhancement at the $p \bar{p}$ mass threshold, dubbed $X(p \bar{p})$, was first observed by BESII in $J / \psi$ radiative decays ${ }^{5}$; this observation was confirmed by BESIII ${ }^{6}$. This enhancement structure was subsequently determined to have spin parity $J^{P}=0^{-}$ by BESIII ${ }^{7}$. The discovery of the $X(1835)$ has stimulated many theoretical speculations concerning its nature. Possible interpretations include a $p \bar{p}$ bound state, a second radial excitation of the $\eta^{\prime}$, and a pseudoscalar glueball. Among these theoretical interpretations, the particularly intriguing one is that the $X(1835)$ and $X(p \bar{p})$ originate from a $p \bar{p}$ bound state.

After the discovery of $X(1835)$ and $X(p \bar{p})$, a number of $X$ states were found by BESIII nearby the $p \bar{p}$ mass threshold. Figure 1 shows the distributions of the measured masses and widths with error bars for those discovered $X$ states. The studied channels are indicated nearby and the corresponding published references are shown in the right corner. Most of them are found in $J / \psi$ radiative decays.

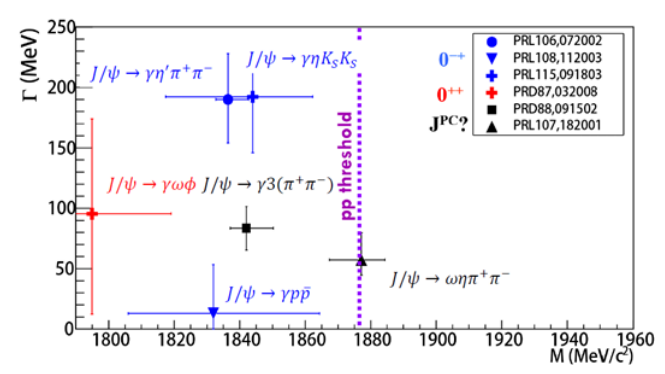

Fig. 1. Distributions of the masses and widths with error bars from some $X$ states discovered by BESIII in low mass regions. For these $X$ states, the discovered channels are also indicated nearby.

According to the above discussions, we have two urgent questions need to be answered. Are these $X$ states from the same resonance ? Why are these $X$ states discovered only by BES(III) ? Confirmation of these $X$ states and precise measurements in different production processes will significantly help us to understand the nature of them.

The first $X Y Z$ state the $X(3872)$, was observed by Belle in $B^{+} \rightarrow X(3872)(\rightarrow$ $\left.J / \psi \pi^{+} \pi^{-}\right) K^{+8}$. Now we know precisely its mass $(3871.69 \pm 0.17) \mathrm{MeV} / c^{2}{ }^{9}$, have a stringent limit on its width (less than $1.2 \mathrm{MeV}$ at $90 \%$ confidence level) ${ }^{10}$ and have a definitive $J^{P C}$ assignment of $1^{++11}$. Later the $X(3872)$ has been observed to decay to several other final states including $J / \psi \gamma, \psi(2 S) \gamma, J / \psi \pi^{+} \pi^{-} \pi^{0}$ and $D^{0} \bar{D}^{* 0}$. Also it has been observed in the decay $B^{0} \rightarrow X(3872) K^{+} \pi^{-}\left(X(3872) \rightarrow J / \psi \pi^{+} \pi^{-}\right)$ 
by Belle, where $B^{0} \rightarrow X(3872) K^{*}(892)^{0}$ is not dominant ${ }^{12}$, and in the process $e^{+} e^{-} \rightarrow \gamma X(3872)$ for the first time with a statistical significance of $6.3 \sigma$ by BESIII using data samples at center-of-mass (c.m.) energies from 4.009 to $4.420 \mathrm{GeV}^{13}$.

Very recently COMPASS experiment observed exclusive production of the $X(3872)$ in the reaction $\mu^{+} N \rightarrow \mu^{+}\left(J / \psi \pi^{+} \pi^{-}\right) \pi^{ \pm} N^{\prime}$ using data collected with incoming muons of $160 \mathrm{GeV} / c$ and $200 \mathrm{GeV} / c$ momentum ${ }^{14}$. Figure 2 (left plot) shows the $J / \psi \pi^{+} \pi^{-}$invariant mass distribution for the $J / \psi \pi^{+} \pi^{-} \pi^{ \pm}$final state for exclusive events with $(-4 \mathrm{GeV}<\Delta E<4 \mathrm{GeV})$ and missing mass above $3 \mathrm{GeV} / c^{2}$, where $\Delta E$ is the difference between the sum of energies of the scattered muon, of the $J / \psi$, and of the three pions in the final state and the beam energy. The statistical significance of the $X(3872)$ signal is $6 \sigma$. Although the $X(3872)$ signal is significant, the corresponding shape of the two pion mass distribution shows disagreement with previous observations, as shown in Fig. 2 (right plot). Further checks with large data samples are needed.
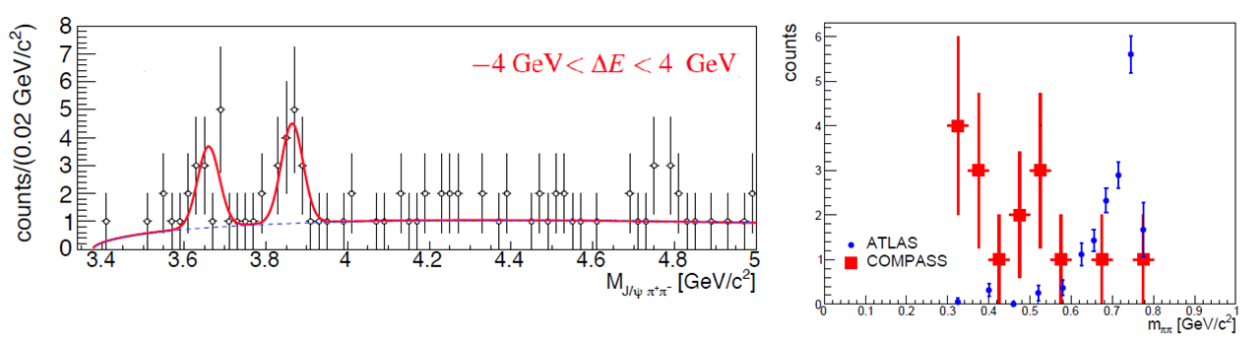

Fig. 2. The $J / \psi \pi^{+} \pi^{-}$and $\pi^{+} \pi^{-}$invariant mass distributions for the $J / \psi \pi^{+} \pi^{-} \pi^{ \pm}$final state for exclusive events with $(-4 \mathrm{GeV}<\Delta E<4 \mathrm{GeV})$ and missing mass above $3 \mathrm{GeV} / c^{2}$.

\section{The $Y$ states}

Among the new charmoniumlike states, there are many vector states with quantum numbers $J^{P C}=1^{--}$that are usually called $Y$ states, like the $Y(4260)$, the $Y(4360)$, and the $Y(4660)$. The $Y$-states show strong coupling to hidden-charm final states in contrast to the vector charmonium states in the same energy region $(\psi(4040), \psi(4160), \psi(4415))$ which couples dominantly to open-charm meson pairs. These $Y$ states are good candidates for new types of exotic particles and stimulated many theoretical interpretations, including tetraquarks, molecules, hybrids, or hadrocharmonia.

In the low mass region, although the $\phi(2170)$ state has been well established, usually also called $Y(2175)$, the differences on the measured mass and width from different experiments are large. The $\phi(2170)$ was first observed by BaBar ${ }^{15}$ in $e^{+} e^{-} \rightarrow \phi f_{0}(980)$ with a mass of $(2175 \pm 10 \pm 15) \mathrm{MeV} / c^{2}$ and a width of $(58 \pm 16 \pm 20)$ $\mathrm{MeV}$. It was subsequently confirmed by Belle in the same process with a mass of $\left(2079 \pm 13_{-28}^{+79}\right) \mathrm{MeV} / c^{2}$ and a width of $\left(192 \pm 23_{-61}^{+25}\right) \mathrm{MeV}^{16}$, and by BESII with a 
mass of $(2186 \pm 10 \pm 6) \mathrm{MeV} / c^{2}$ and a width of $(65 \pm 23 \pm 17) \mathrm{MeV}^{17}$, and by BESIII with a mass of $(2200 \pm 6 \pm 5) \mathrm{MeV} / c^{2}$ and a width of $(104 \pm 15 \pm 15) \mathrm{MeV}^{18}$ in $J / \psi$ hadronic decays. Precise measurements on the $\phi(2175)$ parameters especially its width are important to understand its nature in the future since the width tends to be wide if it is a normal excited $\phi$ state, while to be narrow if it is an $s$-quark counterpart to the $Y(4260)$ (hereafter the $Y(4260)$ is denoted as the $Y(4220)$ since the updated results indicate the lower mass).

In 2013, BESIII reported the cross section measurement of $e^{+} e^{-} \rightarrow \pi^{+} \pi^{-} h_{c}$ at 13 c.m. energies from 3.9 to $4.2 \mathrm{GeV}^{19}$. In 2014, BESIII reported the cross section measurement of $e^{+} e^{-} \rightarrow \omega \chi_{c 0}$ at 9 c.m. energies from 4.21 to $4.42 \mathrm{GeV}$. Assuming a single resonance, BESIII reported a resonant structure with the mass of $(4230 \pm 8 \pm 6) \mathrm{MeV} / c^{2}$ and width of $(38 \pm 12 \pm 2) \mathrm{MeV}$, and the statistical significance is more than $9 \sigma^{20}$. Later BESIII updated the measurements to the above two processes with higher energy data up to $4.6 \mathrm{GeV}$ included ${ }^{21,22}$. In the $e^{+} e^{-} \rightarrow \pi^{+} \pi^{-} h_{c}$ mode, besides the $Y(4220)$, a new structure the $Y(4390)$ was observed with $M=(4391.6 \pm 6.3 \pm 1.0) \mathrm{MeV} / c^{2}$ and $\Gamma=(139.5 \pm 16.1 \pm 0.6) \mathrm{MeV}^{21}$.

Recently, BESIII reported a precise measurement of $e^{+} e^{-} \rightarrow \pi^{+} \pi^{-} J / \psi$ cross sections at c.m. energies from 3.77 to $4.60 \mathrm{GeV}$ using data samples with an integrated luminosity of $9 \mathrm{fb}^{-1} 23$. While the nature of the events at around $4 \mathrm{GeV}$ is still ambiguous, the dominant resonant structure $Y(4220)$ was found to have a mass of $(4222.0 \pm 3.1 \pm 1.4) \mathrm{MeV} / c^{2}$ and a width of $(44.1 \pm 4.3 \pm 2.0) \mathrm{MeV}$. In addition, a new resonance with a mass of around $4.32 \mathrm{GeV} / c^{2}$ is needed to describe the high precision data.

BESIII also reported a measurement of $e^{+} e^{-} \rightarrow D^{0} D^{*-} \pi^{+}+$c.c. cross sections at c.m. energies from 4.05 to $4.60 \mathrm{GeV}$ with a significant improvement over the previous measurement at Belle. Two resonant structures $Y(4220)$ and $Y(4390)$ were observed over a smoothly increasing non-resonant term which can be parametrized with a three-body phase space amplitude.

Considering the $Y(4220)$ was observed in all the above four channels, the authors in Ref. ${ }^{24}$ did a combined fit to measure the resonant parameters of the $Y(4220)$. The determined mass and width are $M=(4219.6 \pm 3.3 \pm 5.1) \mathrm{MeV} / c^{2}$ and $\Gamma=$ $(56.0 \pm 3.6 \pm 6.9) \mathrm{MeV}$. The lower limit of the $Y(4220)$ leptonic decay width of around $30 \mathrm{eV}$ was also extracted, which is close to the prediction from LQCD for a hybrid vector charmonium state ${ }^{25}$.

BESIII studied the process $e^{+} e^{-} \rightarrow \pi^{+} \pi^{-} \psi(2 S)$ using $5.1 \mathrm{fb}^{-1}$ of data collected at 16 c.m. energy points from 4.008 to $4.600 \mathrm{GeV}^{26}$. The measured Born cross sections are consistent with previous results, but with much improved precision. A fit to the cross section shows contributions from the $Y(4220)$ and $Y(4360)$ with the measured $M=(4209.5 \pm 7.4 \pm 1.4) \mathrm{MeV} / c^{2}$ and $\Gamma=(80.1 \pm 24.6 \pm 2.9)$ for the $Y(4220)$, and $M=(4383.8 \pm 4.2 \pm 0.8) \mathrm{MeV} / c^{2}$ and $\Gamma=(84.2 \pm 12.5 \pm 2.1) \mathrm{MeV}$ for the $Y(4360)$. It means another new decay mode of $Y(4220) \rightarrow \pi^{+} \pi^{-} \psi(2 S)$ was observed with a statistical significance of $5.8 \sigma$. 


\section{The $Z$ states}

The BESIII and Belle experiments have observed a charged charmoniumlike state $Z_{c}(3900)$ in $e^{+} e^{-} \rightarrow Y(4220) \rightarrow \pi^{+} \pi^{-} J / \psi^{27,28}$, and subsequently, several similar structures were reported by the BESIII experiment. These observations inspired extensive discussions of their nature, and the dominant interpretation is four-quark states or molecule states. The neutral partners of these charmoniumlike states were also observed, which indicate that each of them is an isotriplet and hint of a new hadron spectroscopy.

The spin and parity of the $Z_{c}^{ \pm}(3900)$ state are determined to be $J^{P}=1^{+}$with a statistical significance larger than $7 \sigma$ over other quantum numbers in a partial wave analysis of the process $e^{+} e^{-} \rightarrow \pi^{+} \pi^{-} J / \psi$ by BESIII using a data sample of $1.92 \mathrm{fb}^{-1}$ accumulated at $\sqrt{s}=4.23$ and $4.26 \mathrm{GeV}^{29}$. When parametrizing the $Z_{c}^{ \pm}(3900)$ with a Flatté-like formula, its pole mass and width are determined to be $(3881.2 \pm 4.2 \pm 52.7) \mathrm{MeV} / c^{2}$ and $(51.8 \pm 4.6 \pm 36.0) \mathrm{MeV}$. Figure 3 shows projections of the simultaneous fit results at $\sqrt{s}=4.23$ and $4.26 \mathrm{GeV}$. If the $Z_{c}^{ \pm}$ is parametrized as a constant-width BW function, the simultaneous fit told us that the BW parametrization is disfavored with a significance of $6.6 \sigma$. The contributions from $Z_{c}^{ \pm}(4020)$ are also searched for, but no significant signals are observed.

A search for the exclusive production of the $Z_{c}^{ \pm}(3900)$ in the reaction $\mu^{+} N \rightarrow$ $\mu^{+} Z_{c}^{ \pm}(3900) N \rightarrow \mu^{+} J / \psi \pi^{ \pm} N \rightarrow \mu^{+} \mu^{+} \mu^{-} \pi^{ \pm} N$ has been performed by COMPASS using the data cover the range from $7 \mathrm{GeV}$ to $19 \mathrm{GeV}$ in the c.m. energy of the photon-nucleon system ${ }^{30}$. The data were obtained scattering positive muons of
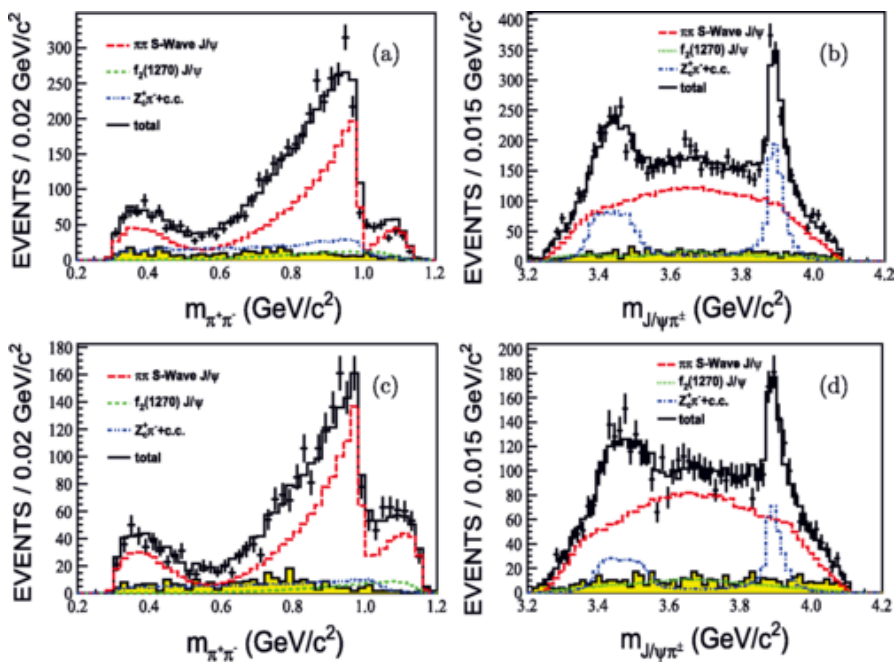

Fig. 3. Projections to $m\left(\pi^{+} \pi^{-}\right)$(a),(c) and $m\left(J / \psi \pi^{ \pm}\right)(\mathrm{b}),(\mathrm{d})$ of the fit results with $J^{P}=1^{+}$ for the $Z_{c}^{ \pm}(3900)$ at $\sqrt{s}=4.23$ (a),(b) and $4.26 \mathrm{GeV}(\mathrm{c}),(\mathrm{d})$. The points with error bars are data, and the black histograms are the total fit results including backgrounds. The shaded histogram denotes backgrounds. The contributions from fitted components are also indicated in the plots. 
$160 \mathrm{GeV} / c$ or $200 \mathrm{GeV} / c$ momentum off solid ${ }^{6} \mathrm{LiD}$ or $\mathrm{NH}_{3}$ targets. The mass spectrum for $J / \psi \pi^{ \pm}$events is shown in Fig. 4, where it does not exhibit any statistically significant resonant structure around $3.9 \mathrm{GeV} / c^{2}$. An upper limit for the ratio $\mathcal{B}\left(Z_{c}^{ \pm}(3900) \rightarrow J / \psi \pi^{ \pm}\right) \times \sigma\left(\gamma N \rightarrow Z_{c}^{ \pm}(3900) N\right)$ of $3.7 \times 10^{-3}$ was established at the confidence level of $90 \%$.

In Belles updated result of $e^{+} e^{-} \rightarrow \pi^{+} \pi^{-} \psi(2 S)$ with full data samples, an evidence for a new $Z_{c}^{ \pm}$state in the mass spectrum of $\pi^{ \pm} \psi(2 S)$ with a mass around $4.05 \mathrm{GeV} / c^{2}$ was observed with a statistical significance of $3.5 \sigma^{31}$. To confirm this state, BESIII recently studied the process $e^{+} e^{-} \rightarrow \pi^{+} \pi^{-} \psi(2 S)$ and a charged charmoniumlike structure is observed in the $\pi^{ \pm} \psi(2 S)$ invariant mass spectrum for data at $4.416 \mathrm{GeV}^{26}$. A fit with an S-wave BW function yields a mass $M=(4032.1 \pm$ 2.4) $\mathrm{MeV} / c^{2}$, where the error is statistical only. However, there are still unresolved discrepancies between the fit model and data. The width of the intermediate state varies in a wide range for different kinematic regions within the data set. Therefore, no simple interpretation of the data has been found, and a future data sample with larger statistics and more theoretical input will be required to better understand this issue.

A full amplitude analysis of $\Lambda_{b}^{0} \rightarrow J / \psi p \pi^{-}$decays was performed by LHCb with a $3 \mathrm{fb}^{-1}$ data sample at 7 and $8 \mathrm{TeV}^{32}$. Amplitude models for the $\Lambda_{b}^{0} \rightarrow J / \psi p \pi^{-}$ decays are constructed to examine the possibility of exotic hadron contributions. A significantly better description of the data is achieved by including the $P_{c}(4380)^{+}$ and $P_{c}(4450)^{+}$to $J / \psi p$ states, and $Z_{c}^{-}(4200)$ to $J / \psi \pi^{-}$state, previously reported by the Belle Collaboration in $B^{0} \rightarrow J / \psi K^{+} \pi^{-}$decays ${ }^{33}$ ( spin parity $J^{P}=1^{+}$, mass and width of $\left(4196_{-29-13}^{+37+17}\right) \mathrm{MeV} / c^{2}$ and $\left(370 \pm 70_{-132}^{+70}\right) \mathrm{MeV}$, respectively). The data support a model containing all three exotic states, with a significance of more than three standard deviations.

By replacing the $c \bar{c}$ pair with a $s \bar{s}$, it is possible to consider an analogous $Z_{s} \rightarrow \phi \pi$ states in $e^{+} e^{-} \rightarrow \phi \pi^{+} \pi^{-}$, where the initial single pion emission (ISPE) mechanism predicts two charged strangeoniumlike structures, $Z_{s}$, one of which is close to the $K^{*} \bar{K}$ mass threshold with a quite narrow peak and the other is close to the $K^{*} \bar{K}^{*}$

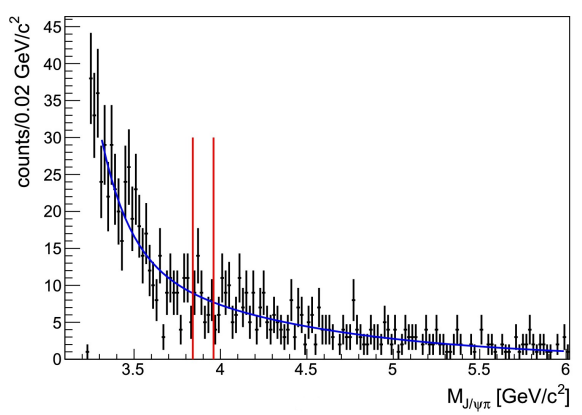

Fig. 4. Mass spectrum of the $J / \psi \pi^{ \pm}$from COMPASS measurement. The fitted function is shown as a line. 
mass threshold with a broad width expected in the $\phi \pi$ invariant mass distribution ${ }^{34}$. Within the ISPE mechanism, the $\phi(2170)$ transits to a $K^{*} \bar{K}^{*}$ pair by emission of a single pion. The low momentum $K^{*}$ and $\bar{K}^{*}$ mesons easily interact with each other and finally transit to $\phi \pi$ by exchanging a $K^{(*)}$ meson ${ }^{34}$. Therefore, it is important to perform an experimental search for the strangeoniumlike structure $Z_{s}$, since its observation may help us to understand the nature of $Z_{c}$ states ${ }^{35}$.

Recently BESIII studied the processes $e^{+} e^{-} \rightarrow \phi \pi^{+} \pi^{-}$and $\phi \pi^{0} \pi^{0}$ to search for the theoretically predicted $Z_{s}$ decaying into $\phi \pi$ using a data sample of $108 \mathrm{pb}^{-1}$ at $\sqrt{s}=2.175 \mathrm{GeV}$. Figure 5 shows the invariant mass distributions of (a) $M\left(\phi \pi_{l}^{ \pm}\right)$ and (b) $M\left(\phi \pi_{l}^{0}\right)$ for the selected $\phi \pi \pi$ candidate events. No signal is observed in the $\phi \pi$ mass spectrum around $1.4 \mathrm{GeV} / c^{2}$, the mass for the $Z_{s}$ predicted with the ISPE. Assuming $Z_{s}$ widths of 0,5 or $10 \mathrm{MeV}$, upper limits on the cross sections of $Z_{s}$ production at the $90 \%$ C.L. are determined.
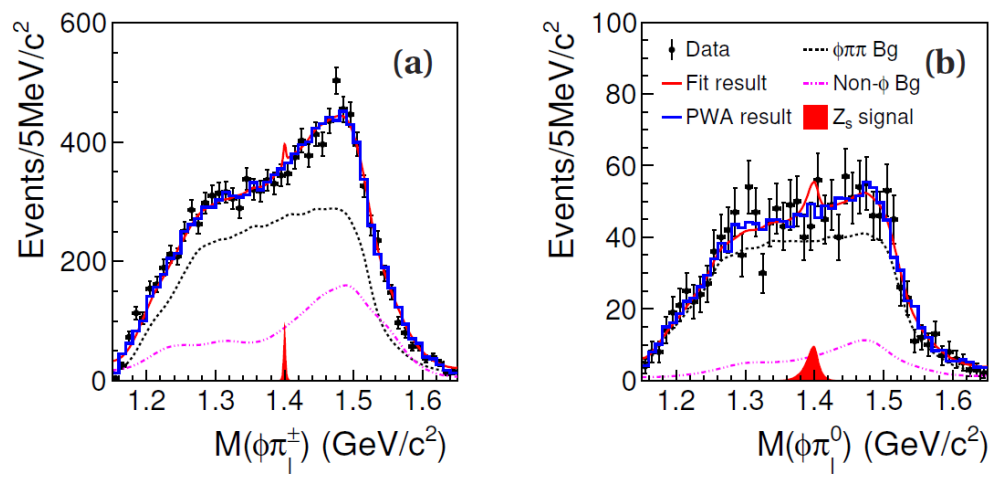

Fig. 5. Invariant mass distributions of (a) $M\left(\phi \pi_{l}^{ \pm}\right)$and (b) $M\left(\phi \pi_{l}^{0}\right)$ for $\phi \pi \pi$ candidate events. The dots with error bars are data, the solid lines are the fit results with the numbers of $Z_{s}$ fixed to the upper limits at the $90 \%$ C.L., the histograms are the projections of the $\phi \pi \pi$ PWA results, the dotted lines are the $\phi \pi \pi$ MC simulated shapes, the dash-dotted lines are non- $\phi$ backgrounds (estimated from $\phi$ mass sideband events), and the shaded histograms are the $Z_{s}$ signal with the production rates fixed to the upper limits (three times for $\phi \pi^{ \pm}$to show the $Z_{s}^{ \pm}$peak clearly).

\section{Conclusion}

There have been great progresses in the study of the $X Y Z$ states, especially Belle and BESIII are still producing more exciting results. However, we found we have more questions to answer. The heavy-flavor, non-standard hadrons have severely challenged existing ideas about the underlying structure of hadrons. The puzzles that they pose have intrigued theorists in both the particle and nuclear physics communities, and experimenters at all of the worlds particle physics accelerator facilities. Further studies along this line may strengthen our understanding of how strong interaction works at low energy and thus a better understanding of the matters around us. Fortunately there are powerful experiments that are currently running and producing important and unique results. 


\section{Acknowledgments}

Supported by National Natural Science Foundation of China (NSFC) under contract No. 11575017; the Ministry of Science and Technology of China under Contract No. 2015CB856701; and the CAS Center for Excellence in Particle Physics (CCEPP).

\section{References}

1. For recent reviews, see, H. X. Chen, W. Chen, X. Liu and S. L. Zhu, Phys. Rept. 639, 1 (2016); N. Brambilla et al., Eur. Phys. J. C 71, 1534 (2011); S. L. Olsen, T. Skwarnicki and D. Zieminska, arXiv:1708.04012.

2. M. Ablikim et al. (BES Collaboration), Phys. Rev. Lett. 95, 262001 (2005).

3. M. Ablikim et al. (BES Collaboration), Phys. Rev. Lett. 106, 072002 (2011).

4. M. Ablikim et al. (BES Collaboration), Phys. Rev. Lett. 115, 091803 (2015).

5. J. Z. Bai et al. (BES Collaboration), Phys. Rev. Lett. 91, 022001 (2003).

6. M. Ablikim et al. (BESIII Collaboration), Chin. Phys. C 34, 421 (2010).

7. M. Ablikim et al. (BESIII Collaboration), Phys. Rev. Lett. 108, 112003 (2012).

8. S.K. Choi et al. (Belle Collaboration), Phys. Rev. Lett. 91, 262001 (2003).

9. C. Patrignani et al. (Particle Data Group), Chin. Phys. C 40, 100001 (2016) and 2017 update.

10. S.K. Choi et al. (Belle Collaboration), Phys. Rev. D 84, 052004 (2011).

11. R. Aaij et al. (LHCb Collaboration), Phys. Rev. Lett. 110, 222001 (2013).

12. A.Bala et al. (Belle Collaboration), Phys. Rev. D 91, 051101(R) (2015).

13. M. Ablikim et al. (BESIII Collaboration), Phys. Rev. Lett. 112, 092001 (2014).

14. M. Aghasyan et al. (COMPASS Collaboration), arXiv:1707.01796.

15. B. Aubert et al. (BABAR Collaboration), Phys. Rev. D 74, 091103(R) (2006).

16. C. P. Shen et al. (Belle Collaboration), Phys. Rev. D 80, 031101 (2009).

17. M. Ablikim et al. (BES Collaboration), Phys. Rev. Lett. 100, 102003 (2008).

18. M. Ablikim et al. (BESIII Collaboration), Phys. Rev. D 91, 052017 (2015).

19. M. Ablikim et al. (BESIII Collaboration), Phys. Rev. Lett. 111, 242001 (2013).

20. M. Ablikim et al. (BESIII Collaboration), Phys. Rev. Lett. 114, 092003 (2015).

21. M. Ablikim et al. (BESIII Collaboration), Phys. Rev. Lett. 118, 092002 (2017).

22. M. Ablikim et al. (BESIII Collaboration), Phys. Rev. D 93, 011102(R) (2016).

23. M. Ablikim et al. (BESIII Collaboration), Phys. Rev. Lett. 118, 092001 (2017).

24. X. Y. Gao, C. P. Shen and C. Z. Yuan, Phys. Rev. D 95, 092007 (2017).

25. Y. Chen, W. F. Chiu, M. Gong, L. C. Gui and Z. Liu, Chin. Phys. C 40, 081002 (2016).

26. M. Ablikim et al. (BESIII Collaboration), Phys. Rev. D 96, 032004 (2017).

27. M. Ablikim et al. (BESIII Collaboration), Phys. Rev. Lett. 110, 252001 (2013).

28. Z. Q. Liu et al. (Belle Collaboration), Phys. Rev. Lett. 110, 252002 (2003).

29. M. Ablikim et al. (BESIII Collaboration), Phys. Rev. Lett. 119, 072001 (2017).

30. C. Adolph et al. (COMPASS Collaboration), Phys. Lett. B 742, 330 (2015).

31. X. L. Wang et al. (Belle Collaboration), Phys. Rev. D 91, 112007 (2015).

32. R. Aaij et al. (LHCb Collaboration), Phys. Rev. Lett. 117, 082003 (2016).

33. K. Chilikin et al. (Belle Collaboration), Phys. Rev. D 90, 112009 (2014).

34. D. Y. Chen et al., Eur. Phys. J. C 72, 2008 (2012).

35. P. L. Liu, S. S. Fang and X. C. Lou, Chin. Phys. C 39, 082001 (2015). 